\title{
Rigidity for closed manifolds with positive curvature
}

\author{
Changyu Xia
}

Received: 19 November 2008 / Accepted: 15 December 2008 / Published online: 6 January 2009

(C) Springer Science+Business Media B.V. 2009

\begin{abstract}
Let $M$ be an $n$-dimensional complete connected Riemannian manifold with sectional curvature $\sec (M) \geq 1$ and $\operatorname{radius} \operatorname{rad}(M)>\pi / 2$. In this article, we show that $M$ is isometric to a round $n$-sphere if for any $x \in M$, the first conjugate locus of $x$ is a single point and if $M$ contains a geodesic loop of length $2 \cdot \operatorname{rad}(M)$. We also show that the same conclusion is true if the conjugate value function at any point of $M$ is a constant function.
\end{abstract}

Keywords Rigidity $\cdot$ Closed manifolds $\cdot$ Sectional curvature $\cdot$ Conjugate locus

Mathematics Subject Classification (2000) $\quad 53 \mathrm{C} 20$

\section{Introduction}

Let $M$ be an $n$-dimensional complete connected Riemannian manifold with sectional curvature $\sec (M) \geq 1$ and denoted by $d$, the distance function on $M$. For a point $x \in M$, the radius of $M$ at $x$ is defined by $\operatorname{rad}(x)=\max _{y \in M} d(x, y)$ and the radius of $M$ is given by $\operatorname{rad}(M)=\min _{x \in M} \operatorname{rad}(x)$. This invariant was introduced by Shiohama and Yamaguchi in [15]. When the radius of $M$ is bigger than $\pi / 2$, Grove and Petersen [11] showed that the volume of $M$ satisfies $C(n) \leq \operatorname{vol}(M) \leq\{\operatorname{rad}(M) / \pi\} \omega_{n}$, where $\omega_{n}$ is the volume of a unit Euclidean $n$-sphere and $C(n)$ is a positive constant depending only on $n$. It has been proved by Wang [17] that if $\operatorname{rad}(M)>\pi / 2$, then the radius of any closed totally geodesic submanifold of $M$ is not less than that of $M$ and, in particular, the length of any closed geodesic of $M$

This work was done while the author was visiting MPI for Mathematics in Leipzig, Germany. The author is very grateful to MPI for Mathematics in Leipzig for its hospitality and CAPES.

C. Xia $(\varangle)$

Departamento de Matemática, Universidade de Brasília, Campus Universitário, 70910-900, Brasília, DF, Brazil

e-mail: xia@mat.unb.br

C. Xia

MPI for Mathematics in the Sciences, Inselstr. 22, 04103 Leipzig, Germany 
is bigger than or equal to $2 \cdot \operatorname{rad}(M)$. Motivated by the above Wang's theorem, we will study in this article the rigidity of positively curved manifolds with large radius by the existence of some special geodesic. Before stating our main result, we fix some notation. Let $M$ be a complete Riemannian manifold. We will always consider geodesics as curves $\gamma$ from some interval say $[0, \mathrm{~b}]$ into $M$, parametrized by arc-length, and we will say that $\gamma$ issues from $x \in M$ if $\gamma(0)=x$. For a point $m \in M$, we denote by $\operatorname{conj}(m)$ the conjugate locus of $M$ at $m$, that is, the set of the first conjugate points to $m$, for all geodesics that start at $p$. A geodesic loop of length $l$ issuing from $m$ is a geodesic $\gamma:[0, l] \rightarrow M$ such that $\gamma(0)=\gamma(l)=m$ and $\gamma(t) \neq m$ for each $t \in(0, l)$. A geodesic loop of length $l$ issuing from $m$ is simple if it is one-to-one on $[0,1)$.

For a point $x$ in $M$, denote by $S_{x} M$ the unit tangent sphere of $M$ at $x$. For any $v \in S_{x} M$, let $\gamma$ be a unit speed geodesic with $\gamma^{\prime}(0)=v$. The conjugate value $c_{v}$ of $v$ is defined to be the first number $r>0$ such that there is a nonzero Jacobi field $J$ along $\gamma$ satisfying $J(0)=J(r)=0$. Set

$$
\operatorname{conj}(x):=\inf _{v \in S_{x} M} c_{v} .
$$

We call $\operatorname{conj}(x)$ the conjugate radius of $M$ at $x$. The conjugate radius of $M$ is defined as $\operatorname{conj}(M)=\inf _{x \in M} \operatorname{conj}(x)$.

Now we can state the main results in this article as follows:

Theorem 1.1 Let $M$ be an $n$-dimensional complete connected Riemannian manifold $M$ with $\sec (M) \geq 1$ and $\operatorname{rad}(M)>\pi / 2$. Assume that for any $x \in M$, the first conjugate locus of $x$ is a single point. If $M$ contains a geodesic loop of length $2 \cdot \operatorname{rad}(M)$, then $M$ is isometric to a round $n$-sphere.

Theorem 1.2 Let $M$ be an $n$-dimensional complete connected Riemannian manifold $M$ with $\sec (M) \geq 1$ and $\operatorname{rad}(M)>\pi / 2$. Assume that for any $x \in M$, the first conjugate value function $c_{x}: S_{x} M \rightarrow R$ is a constant function. Then $M$ is isometric to a round $n$-sphere.

Let $R P^{n}$ be an $n$-dimensional real projective space of sectional curvature 1 . Then, we have $\operatorname{rad}\left(R P^{n}\right)=\pi / 2$, and for any $x \in R P^{n}, \operatorname{conj}(x)=\{x\}$. Also, $R P^{n}$ has many closed geodesics of length $\pi$. From this example, we know that the condition " $\operatorname{rad}(M)>\pi / 2$ " in the above Theorems is essential.

The structure of closed manifolds with positive sectional curvature has been an important topic in global Riemannian geometry. One can find various interesting results about positively curved manifolds, e.g., in [1-3,5,7,9-17,20,21].

\section{Proofs of the results}

Before proving our main results in this article, we list some facts that are needed. Let $M$ be a complete connected Riemannian $n$-manifold satisfying $\sec (M) \geq 1$ and $\operatorname{rad}(M)>\pi / 2$. It follows by using the Toponogov comparison theorem that for any $x \in M$, there exists a unique point $A(x)$ which is at the maximal distance from $x$. The mapping $A: M \rightarrow M$ is easily seen to be continuous (Cf. [11,21]). Observe that $M$ is homeomorphic to $S^{n}$. Thus, we know from the Brouwer fixed point theorem that $A$ is surjective. For a point $x \in M$, we denote by $\operatorname{inj}(x)$ and $\operatorname{cut}(x)$ the injectivity radius and the cut locus of $M$ at $x$, respectively.

Recall that a wiedersehen manifold is a connected simply connected compact Riemannian $n$-manifold $M$ without boundary such that for any $m \in M$ the cut locus of $m$ is a single 
point [8]. It is known that a wiedersehen manifold is isometric to a round sphere of constant curvature $[4,8,18,19]$.

We will prove that the manifolds $M$ in Theorems 1 and 2 are wiedersehen manifolds. Since $M$ is homeomorphic to a sphere, it then suffices to show that the cut-locus of any point of $M$ is a single point.

Proof of Theorem 1.1 Let us first prove the following Lemma.

Lemma 1 Let $m$ be a point of $M$ and let $\eta:[0, b] \rightarrow M$ be a simple geodesic loop issuing from $m$, then $b \geq 2 \cdot \operatorname{rad}(m)$.

Proof of Lemma 1 Let us first show that $b>\pi$. Suppose on the contrary that $b \leq \pi$. Set $\tilde{m}=\eta(b / 2)$ and take $m_{1} \in M$ so that $m=A\left(m_{1}\right)$; then $m_{1} \neq \tilde{m}$. Set $s=d\left(m, m_{1}\right)$ and $t=d\left(\tilde{m}, m_{1}\right)$; then $s>t$. Let $\mu$ be a minimal geodesic from $\tilde{m}$ to $m_{1}$; then we have either

$$
\angle\left(\mu^{\prime}(0), \eta^{\prime}\left(\frac{b}{2}\right)\right) \leq \frac{\pi}{2},
$$

or

$$
\angle\left(\mu^{\prime}(0),-\eta^{\prime}\left(\frac{b}{2}\right)\right) \leq \frac{\pi}{2} .
$$

We can then apply the Toponogov comparison theorem to the hinge $\left(\mu,\left.\eta\right|_{[0, b / 2]}\right)$ or $\left(\mu,\left.\eta\right|_{[b / 2, b]}\right)$ to obtain

$$
0>\cos s \geq \cos \frac{b}{2} \cos t
$$

This is a contradiction since $t<s$ and $b \leq \pi$. Thus, we have $b>\pi$. Set $r=\operatorname{rad}(m)=$ $d(m, A(m))$ and suppose that $b<2 r$. Let $u=d(\tilde{m}, A(m))$ and take a minimal geodesic $\lambda$ from $\tilde{m}$ to $A(m)$. Using the Toponogov comparison theorem to the hinges $\left(\lambda,\left.\eta\right|_{[b / 2, b]}\right)$, we have

$$
0>\cos r \geq \cos \frac{b}{2} \cos u,
$$

which gives $\cos u>0$. It follows from $b / 2<r$ that

$$
\cos u \cos \frac{b}{2}>\cos u \cos r \text {. }
$$

Introducing (2.2) into (2.1), we get

$$
\cos r(1-\cos u)>0 .
$$

This is a contradiction and completes the proof of Lemma 1.

Let $l=\operatorname{rad}(M)$ and let $\gamma:[0,2 l] \rightarrow M$ be a geodesic loop issuing from $p$. Then $\gamma$ is simple. Otherwise, we can find two distinct numbers $l_{1}, l_{2} \in(0,2 l)$ such that $\gamma\left(l_{1}\right)=\gamma\left(l_{2}\right)$ and that $\left.\gamma\right|_{\left[l_{1}, l_{2}\right]}$ is simple. It then follows from Lemma 1 that

$$
l_{2}-l_{1}=\text { the length of }\left.\gamma\right|_{\left[l_{1}, l_{2}\right]} \geq 2 \cdot \operatorname{rad}(M)=2 l,
$$

which contradicts to the fact that $2 l>l_{2}-l_{1}$. We claim that $\gamma(l)=A(p)$ and that $\left.\gamma\right|_{[0, l]}$ and $\left.\gamma\right|_{l, 2 l]}$ are minimizing geodesics. In fact, since

$$
d(p, \gamma(l)) \leq \operatorname{rad}(M) \leq \operatorname{rad}(p)=d(p, A(p)),
$$


in order to prove the above claim, it suffices to show that $\gamma(l)=A(p)$. Suppose by contradiction that $\gamma(l) \neq A(p)$. Set $t=d(\gamma(l), A(p)), r=d(p, A(p))$; then $t>0$. Take a minimal geodesic $\alpha$ from $\gamma(l)$ to $A(p)$; then we have either

$$
\angle\left(\gamma^{\prime}(l), \alpha^{\prime}(0)\right) \leq \frac{\pi}{2}
$$

or

$$
\angle\left(-\gamma^{\prime}(l), \alpha^{\prime}(0)\right) \leq \frac{\pi}{2} .
$$

We assume without loss of generality that $L\left(-\gamma^{\prime}(l), \alpha^{\prime}(0)\right) \leq \pi / 2$. Applying the Toponogov comparison theorem to the hinge $\left(\left.\gamma\right|_{[0, l]}, \alpha\right)$, we get

$$
\begin{aligned}
0>\cos r & \geq \cos t \cos l+\sin t \sin l \cos \angle\left(-\gamma^{\prime}(l), \alpha^{\prime}(0)\right) \\
& \geq \cos t \cos l
\end{aligned}
$$

which gives $\cos t>0$ since $l>\pi / 2$. It then follows from $l \leq r$ that

$$
\cos t \cos l \geq \cos t \cos r
$$

which, combining with (2.4), gives

$$
\cos r(1-\cos t) \geq 0 .
$$

This is a contradiction. Thus, our claim is true.

From $\sec (M) \geq 1,2 l>\pi$, the above claim and the Rauch comparison theorem (see [6, p. 218] we know that there exists an $h \in[l, 2 l)$ such that $\gamma(h)$ is the first conjugate point of $\gamma(0)$ along $\left.\gamma\right|_{[0, h]}$. Similarly, if we consider the reversed curve $\tilde{\gamma}$ of $\gamma$, that is, $\tilde{\gamma}(t)=\gamma(2 l-t), t \in[0,2 l]$, then there exists a $\tilde{h} \in[l, 2 l)$ such that $\gamma(\tilde{h})$ is the first conjugate point of $\tilde{\gamma}(0)$ along $\left.\tilde{\gamma}\right|_{[0, \tilde{h}]}$. Since $\operatorname{conj}(\gamma(0))$ is a single point, this forces $h=\tilde{h}=l$, that is, $\operatorname{conj}(\gamma(0))=\{\gamma(l)\}$.

Set $\tilde{l}=\operatorname{inj}(p)$; then $\tilde{l} \leq l$. Take a point $\tilde{p} \in \operatorname{cut}(p) \operatorname{such}$ that $\operatorname{inj}(p)=d(p, \tilde{p})$. By Proposition 2.12 in [6, p. 274], we know that either

(i) there exists a minimizing geodesic $\beta$ from $p$ to $\tilde{p}$ along which $\tilde{p}$ is conjugate to $p$; or

(ii) there exist exactly two minimizing geodesics $\beta_{1}$ and $\beta_{2}$ from $p$ to $\tilde{p}$ satisfying $\beta_{1}^{\prime}(\tilde{l})=$ $-\beta_{2}^{\prime}(l)$.

If statement (i) holds, then $\tilde{p} \in \operatorname{conj}(\gamma(0))$ and so $\tilde{p}=\gamma(l)$. On the other hand, if statement (ii) holds, then $\beta_{1} \cup \beta_{2}$ is a geodesic loop and so one knows from Lemma 1 that $\tilde{l} \geq \operatorname{rad}(p)$. It follows that $\operatorname{inj}(p)=\tilde{l}=l=\operatorname{rad}(p)$. Also, one concludes from the above claim that $\beta_{1}(l)=A(p)$. Consequently, we have $\operatorname{cut}(p)=\{A(p)\}$. Since $A(p)$ is the unique point that is at the maximal distance from $p$, it follows that any geodesic $\sigma:[0, l] \rightarrow M$ issuing from $p$ is minimizing and satisfies $\sigma(l)=A(p)$. Set $A(p)=q$ and let $\gamma_{1}:[0, l] \rightarrow M$ be the geodesic with $\gamma_{1}(0)=p$ and $\gamma_{1}^{\prime}(0)=-\gamma^{\prime}(0)$; then $\gamma_{1}$ is minimizing, $\gamma_{1}(l)=q$ and by using a new parametrization, $\left.\gamma\right|_{[0, l]} \cup \gamma_{1}$ is a geodesic loop of length $2 l$ issuing from $q$. It then follows from Lemma 1 that $2 l \geq 2 \cdot \operatorname{rad}(q)$. On the other hand, we have $\operatorname{rad}(q) \geq d(q, p)=l$. Thus, $\operatorname{rad}(q)=l$ and one concludes by using the same arguments as in the proof of the above claim that $A(q)=p$. Also, we know from the above discussions in the proof of $\operatorname{inj}(p)=l$ that $\operatorname{inj}(q)=l$. It follows that if $\delta:[0,2 l] \rightarrow M$ is a geodesic issuing from $p$ then it must satisfy $\delta(l)=q, \delta(2 l)=p$. Let us prove that such a $\delta$ is smooth at $p$. Suppose on the contrary that $\delta$ is not smooth at $p$. Take an $\epsilon>0$ so that $2 l \geq \pi+2 \epsilon$. Since $\left.\delta\right|_{[0, l]}$ is minimizing, we know that $\left.\delta\right|_{[\epsilon, l]}$ has no conjugate points. Observe that the 
length of $\left.\delta\right|_{[\epsilon, 2 l-\epsilon]}$ is no less than $\pi$. We know from the Rauch comparison theorem that there exists an $\epsilon_{1} \in(l, 2 l-\epsilon]$ such that $\delta\left(\epsilon_{1}\right)$ is the first conjugate point of $\delta(\epsilon)$ along $\left.\delta\right|_{[\epsilon, 2 l-\epsilon]}$. Let $\tilde{\delta}:[0,2 l] \rightarrow M$ be a geodesic such that $\tilde{\delta}(0)=p$ and $\tilde{\delta}^{\prime}(0)=-\delta^{\prime}(0)$; then $\tilde{\delta}_{[0, l]}$ and $\left.\tilde{\delta}\right|_{[l, 2 l]}$ are minimizing geodesics and $\delta \cap \tilde{\delta}=\{p, q\}$. Observe that $\left.\left.\delta_{1} \equiv \delta\right|_{[0, \epsilon]} \cup \tilde{\delta}\right|_{[0,2 l-2 \epsilon]}$ is a smooth geodesic of length $\geq \pi$ from $\delta(\epsilon)$ to $\delta(2 l-2 \epsilon)$. It follows that there exists an $\epsilon_{2} \in(0,2 l-2 \epsilon]$ such that $\tilde{\delta}\left(\epsilon_{2}\right)$ is the first conjugate point of $\delta(\epsilon)$ along the geodesic $\delta_{1}$. Since $\operatorname{conj}(\delta(\epsilon))$ is a single point, we know that $\tilde{\delta}\left(\epsilon_{2}\right)=\delta\left(\epsilon_{1}\right)$. This is a contradiction since $\delta\left(\epsilon_{1}\right) \notin\{p, q\}$ and $\delta \cap \tilde{\delta}=\{p, q\}$. Thus $\delta$ is smooth at $p$. Since the length of $\delta$ is $2 \cdot \operatorname{rad}(M)$, we conclude by using the similar arguments as in the proof of $\operatorname{cut}(p)=\{A(p)\}$ that for any $z \in \delta, \operatorname{cut}(z)=\{A(z)\}$. Now we fix a point $u \notin \gamma$ and let us prove that $\operatorname{cut}(u)=\{A(u)\}$. Set $t=d(x, u)$ and take a minimizing geodesic $c$ from $p$ to $u$. From the above discussions, we know that $c$ can be extended to a closed geodesic (still denoted by $c$ ) $c:[0,2 l] \rightarrow M$ satisfying $c(0)=c(2 l)=p$ and $c(l)=A(p)$. From the above arguments, we know that $\operatorname{cut}(u)=\{A(u)\}$. Consequently, $M$ is a wiedersehen manifold and so is isometric to a round sphere of constant curvature. This completes the proof of Theorem 1.

Proof of Theorem 2 Fix a point $p \in M$, let us prove that $\operatorname{cut}(p)$ is a single point. Take a point $q \in \operatorname{cut}(p)$ so that $s \equiv \operatorname{inj}(p)=d(p, q)$. Then we have as in the proof of Theorem 1 that either

(iii) there exists a minimizing geodesic $\alpha$ from $p$ to $q$ along which $q$ is conjugate to $p$; or

(iv) there exist exactly two minimizing geodesics $\alpha_{1}$ and $\alpha_{2}$ from $p$ to $q$ satisfying $\alpha_{1}^{\prime}(s)=$ $-\alpha_{2}^{\prime}(s)$.

Set $l=d(p, A(p))$; then $l \geq s$. If statement (iii) holds, then for any $v \in S_{p} M, c_{p}(v)=s$. Let $\gamma:[0, l] \rightarrow M$ be a minimizing geodesic from $p$ to $A(p)$. From $c_{p}\left(\gamma^{\prime}(0)\right)=s$, we know that $\gamma(s)$ is the first conjugate point of $p$ along $\gamma$. Observe that $\gamma$ is minimal and so $\left.\gamma\right|_{[0, l)}$ has no conjugate points. It follows that $s=l$. If (iv) holds, then $\alpha_{1} \cup \alpha_{2}$ is a geodesic loop at $p$ and so $2 s=$ the length of $\alpha_{1} \cup \alpha_{2} \geq 2 l$ by Lemma 1 . Thus in both cases, we have $s=l$, that is, $d(p, q)=d(p, A(p))$. We conclude therefore that $q=A(p)$ since $A(p)$ is the unique point that is at the maximal distance from $p$. Now for any $z \in \operatorname{cut}(p)$, it then follows from $d(p, z) \geq d(p, q)=d(p, A(p))$ that $z=A(p)$. Consequently, we have $\operatorname{cut}(p)=\{A(p)\}$. This completes the proof of Theorem 2 .

Remark Motivated by Theorem 1, it is natural to study the following problem.

Let $M$ be an $n$-dimensional complete connected Riemannian manifold $M$ with $\sec (M) \geq 1$ and $\operatorname{rad}(M)>\pi / 2$. Assume that $M$ contains a closed geodesic of length $2 \cdot \operatorname{rad}(M)$. Is it true that $M$ is isometric to a round $n$-sphere?

\section{References}

1. Abresch, U., Meyer, T.W.: Pinching below $\frac{1}{4}$, injectivity radius, and conjugate radius. J. Differential Geom. 40, 643-691 (1994)

2. Abresch, U., Meyer, T.W.: A sphere theorem with a pinching constant below $\frac{1}{4}$. J. Differential Geom. 44(2), 214-261 (1996)

3. Abresch, U., Meyer, T.W.: Injectivity radius estimates and sphere theorems. In: Grove, K., et al. (eds.) Comparison Geometry (Berkeley, CA, 1993-94), pp. 1-47. Mathematical Sciences Research Institute Publishing, vol.30. Cambridge University Press, Cambridge (1997)

4. Besse, A.L.: Manifolds All of Whose Geodesics are Closed, Appendix D and Appendix E, Ergebnisse der Mathematik und ihrer Grenzgebiete, vol.93. Springer, Berlin (1978)

5. Cheeger, J., Ebin, D.: Comparison theorems in Riemannian Geometry, North-Holland, New York, (1975) 
6. do Carmo, M.P.: Riemannian Geometry. Bikhauser, Boston (1993)

7. Fukaya, K.: Metric Riemannian geometry Handbook of Differential Geometry, vol. II . pp. 189-313. Elsevier/North-Holland, Amsterdam (2006)

8. Green, L.W.: Aufwiedersehnsflachen. Ann. Math. 78(2), 289-299 (1963)

9. Gromoll, D., Grove, K.: A generalization of Berger's rigidity theorem for positively curved manifolds. Ann. Sci. Éc. Norm. Sup. 20, 227-239 (1987)

10. Grove, K.: Critical point theory for distance functions. In: Differential geometry: Riemannian geometry (Los Angeles, CA, 1990), pp. 357-385. Proceedings of the Symposium on Pure Mathematics, vol. 54(3). American Mathematical Society, Providence, RI, (1993)

11. Grove, K., Petersen, P.: Volume comparison á la Aleksandrov. Acta Math. 169, 131-151 (1992)

12. Grove, K., Petersen, P.: A radius sphere theorem. Invent. Math. 112, 577-583 (1993)

13. Grove, K., Shiohama, K.: A generalized sphere theorem. Ann. Math. 106, 201-211 (1977)

14. Micallef, M., Moore, J.D.: Minimal two-spheres and the topology of manifolds with positive curvature on totally isotropic two-planes. Ann. Math. 127, 199-227 (1988)

15. Shiohama, K., Yamaguchi, T.: Positively curved manifolds with restricrted diameters. In: Shiohama, K. (ed.) Geometry of Manifolds. Perspectives in Mathematics, vol.8, pp.345-350. Academic press, New York (1989)

16. Toponogov, V.A.: computation of the length of a closed geodesic on a convex surface. Dokl. Akad. Nauk. SSSR 124, 282-284 (1959)

17. Wang, Q.: On the geometry of positively curved manifolds with large radius. Illinois J. Math. 48, 89-96 (2004)

18. Yang, C.T.: Odd-dimensional wiedersehen manifolds are spheres. J. Differ. Geom. 15, 91-96 (1980)

19. Yang, C.T.: On the Blaschke conjecture. Seminar on Differential Geometry, Annals of Mathematical Studies, vol. 102, pp. 159-171. Princeton University Press, Princeton, NJ (1982)

20. Xia, C.: Some applications of critical point theory of distance functions on Riemannian manifolds. Compos. Math. 132, 49-55 (2002)

21. Xia, C.: A round sphere theorem for positive sectional curvature. Compos. Math. 142, 1327-1331 (2006) 